\title{
The Research and Application of Virtual Reality (VR) Technology in Agriculture Science
}

\author{
Feng Yu ${ }^{1}$, Jun-feng Zhang ${ }^{1}$, Yousen Zhao ${ }^{2}$, Ji-chun Zhao ${ }^{1, *}$, Cuiping Tan $^{1}$, \\ and Ru-peng Luan ${ }^{1}$ \\ ${ }^{1}$ Institute of Information on Science and Technology of Agriculture, \\ Beijing Academy of Agriculture and Forestry Sciences, Beijing, China, 100097, \\ Tel.:86- 010-51503135 \\ yufaari.ac.cn \\ ${ }^{2}$ The Information Center of Beijing Municipal Bureau of Agriculture, Beijing, China
}

\begin{abstract}
The construction of multi-media information resources of crops and livestock is discussed for interactive three-dimensional animation education in the paper. The animation technology, three-dimensional visualization technology and digital entertainment pattern are combined with modern agricultural science technology knowledge and the production process. Based on the application of virtual reality technology, animation and digital entertainment form, including crops, such as corn, cucumber, etc., livestock such as pig farms, chicken farms, etc., farmers and young people are more willing to acceptable, easy to understand and easy to master knowledge of agriculture, and the VR technology is researched in the text.
\end{abstract}

Keywords: Virtual Reality, three-dimensional visualization, animation technology.

\section{Introduction}

Virtual reality, virtual reality (VR) as early as 1965 by the Ivan Sutherland and others raised, development in the 1980s and 1990s, is considered a new technology. VR is a comprehensive integrated technology, computer graphics, human - computer interaction, sensor technology, artificial intelligence, it is used in areas such as computer stereo vision, hearing, olfaction, etc. enable the user through the appropriate device, naturally on the virtual world experience and interaction, enables users to produce a kind of right. At present, its application has been involved in scientific research, education and training, engineering design, commercial, military, medical, film and television, and so on many areas, is recognized as experts in the 21 st century society one of the development of technology.

\section{The Meaning of VR}

Virtual reality system in theory should have the following four aspects of meaning: (1) Immersion. Participants in the integration to create a virtual computer environment,

\footnotetext{
* Corresponding author.
} 
and do not feel the external environment itself. (2) Multi-Sensory. Virtual reality technology ideal person should have all the perceptual features, including visual, auditory, force, tactile perception, including taste, smell, such as perception. Due to restrictions on sensor technology, the current virtual reality technology can offer is limited to visual perception, auditory, force, tactile and so on. (3) Interactive. Participants in a virtual environment with a variety of objects are participating in Circumstances. For example, participants can hand-catching virtual objects, when the hands are holding something of the feeling and can feel the hardness and the weight of objects, the object was captured with the hand movements of the mobile. (4) autonomy. Autonomy refers to a virtual environment to follow the objective law of the material performance. System in their own object models and rules are in accordance with the requirements of computer users in their own campaigns.

\section{The Structure of VR Technology}

Virtual Reality refers to the use of a computer-generated simulation of the environment, and through a variety of special equipment allows the user inputs to the environment, the user directly with the natural environment technology interaction.VR technology allows users to use people's natural skills of virtual objects in the world to inspect or operate, while providing visual, hearing, touch and other natural and intuitive perception of the real-time. Visibility, virtual reality gratitude is made up of people, machinery, and environment three parts:

The environment is generated by a computer can provide a visual, hearing, touch, smell and taste sensory world, which can be a true realization of the real world, and can also be a virtual concept world.

Machine is the computer systems and three-dimensional interactive equipment, which used a helmet with a three-dimensional, data gloves, three-dimensional mouse users, such as wearing the device. In reality there are settings in the environmentsensing devices, such as cameras, various sensors.

People are simulated environment participant which is dealt by the computer processing the action data that can bring immersive feel.

A typical virtual reality system is mainly composed by the 6 parts: computergenerated virtual reality and processes systems, software application systems, and virtual reality related to the theory and technology, input and output man-machine interface devices, users and databases. VR system architecture as shown in Figure 1:

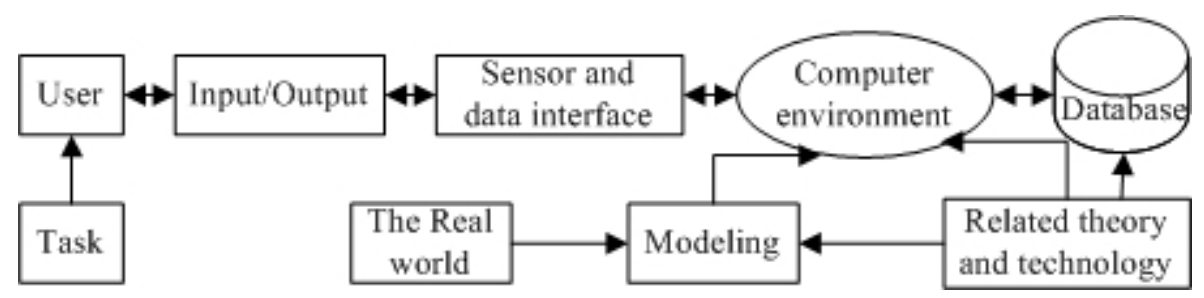

Fig. 1. The structure of VR system 
In the virtual reality system, the computer is responsible for generating the virtual world and the realization of human-computer interaction. As the virtual world is a high degree of complexity, making deep into the virtual world a large amount of computing, therefore, virtual reality has higher requirements for the computer system configuration. This shows that the computer is the heart of VR systems. For the realization of the virtual world and the natural interaction, it is necessary to adopt special input and output devices to identify various forms of user input and generate real-time feedback to achieve a dialogue between man and computer. Theories and technologies related to the establishment of a virtual environment to provide the necessary theory, technology and so on. The function include: the virtual world of geometric objects model, physical model, behavior model, three-dimensional virtual stereo generation, model management techniques and real-time display technology, virtual world database, such as the establishment and management of several parts. Mainly used in the virtual world database storing the virtual world of information in all aspects of objects. Eventually the virtual reality systems are provided to user applications. The key technologies of Virtual Reality involved are: the scene of large-scale three-dimensional data modeling techniques; dynamic real-time three-dimensional vision, hearing, such as generating technologies; three-dimensional positioning; threedimensional interactive software and system integration technology.

\section{VR Applications in Agriculture}

Virtual agriculture is a virtual reality technology applied to agricultural, which refers to the computer in the implementation of substance in virtual soil adsorption, emissions, the migration process, animal and plant growth process, the result of expression, assimilation, alienation, and so on. Researchers explore various stress conditions, human intervention conditions on the role of these procedures. Virtual technology applied to agriculture in the field of scientific research, teaching, agricultural resources, planning, production, circulation of goods and agricultural machinery design and manufacturing, etc.

\subsection{Virtual Plant}

Virtual plant is, following the development of information technology in the past 20 years, rapidly developed areas of research, widely used in the agronomy, forestry, ecology, remote sensing multi-fields, etc. virtual plant is to use VR technology in 3D simulation of plants and plant morphology of the growth process. Using virtual plant technology, on the computer screen design, simulation of the whole plant crops or even the whole group of plants throughout the life cycle, do not have to take a long time crops, it saves time, manpower and money. Such as virtual fertilization and fruit pruning, breeding of plant type analysis, etc. You can implement the implementation of the crop of dynamic growth process on your computer; obtain the various parameters of dynamic data, instead of traditional agriculture situation which is difficult to quantitative study, which can provides reference data for the intelligence agriculture and delicate operation agriculture. You can implement a plant of threedimensional structure of visualization, provides methods and means to structure and 
research on crop Physiology and ecology of the relationship between crop plant type design and genotypes, etc. so far, virtual plant research including plant parts and root portion.

\subsection{Virtual Reality Technology Application in Agricultural Machinery Design and Manufacture}

Due to the characteristics of agricultural machinery design, in the development process, use the computer to draw a lot of new products and parts must be in the manufacturing process before you can learn about the product performance. When performance is not required to meet the requirements, product design will be modified, resulting in product design cycle length, wasted property, it is difficult to adapt to changing market demands. Virtual reality three-dimensional modeling technology is to improve the level of agricultural machinery product design an effective way. Three-dimensional technology can display the image of intuitive product appearance and difficult to quantify the expression of two-dimensional works view and the key parts of complex surface, allowing designers make it easier to exam design products, but also makes it easier to understand the product characteristics. Engineer in product processing before, comprehensive evaluation virtual products that could make him feel the future of the product - related performance and feasibility of providing decision making and optimizing ahead of the implementation plan.

\subsection{Virtual Reality Technology Application in Teaching and Agriculture Popularization}

The application virtual reality technology is the multimedia and interactive computer technology, combining the results of education in creative and effectiveness have been breakthroughs. students see crop growth conditions dynamic process from any angle, as they move the mouse and key, they can see, hear and control over their observations on the screen angle, who roaming in which obtained better results than the traditional way. We set up virtual farm with virtual plant model technology, which enables farmers on the computer management virtual crops and planting virtual farmland, by changing environmental conditions and crop cultivation practices, they visually observation on the status changes in crop growth. virtual technology applied to the achievement of agricultural science and technology promotion, farmers will make it easier to understand and grasp advanced agricultural land management techniques, so the process of teaching have become more and more intuitive, vivid image, which greatly enhances teaching effectiveness and increases their learning interest.

\section{Conclusion}

Above all, virtual reality technology improve the using efficiency of agricultural production, which can improve the efficiency of agricultural resources comprehensive utilization, which can simulation agricultural product market transactions and agricultural production management, which can realize the agricultural and technical education, training, research etc. Virtual reality technology can permeate various fields of 
agricultural production. Therefore, It has great significance that virtual reality technology application was researched in agriculture, It is extremely complex and very long cycle life science research on space-time quantitative analysis of coordinate system, which can greatly reduce the research cycle and also can get direct experimental results. However, virtual reality technology application have a scientific method, as do the guidance of an effective supplementary means of scientific research in order to better promote the development of agriculture.

\section{References}

Baomin, Y.: The application of Distributed VR environment in composes campaign body exercise simulating. Computer Simulation 14(4), 17-201 (1997)

Pemmaraju, S.V., Pirwani, I.A.: Good Quality Virtual Realization of Unit Ball Graphs. In: Arge, L., Hoffmann, M., Welzl, E. (eds.) ESA 2007. LNCS, vol. 4698, pp. 311-322. Springer, Heidelberg (2007)

Shen, W.j., Zhao, C.j., Shen, Z.r., Guo, X.y.: Virtual Reality Technology and Its Application in Agriculture. Research of Agricultural Modernization, 9 (2002)

http://www.sgi.com/products/software/opengl/tech_info.html

http://en.wikipedia.org/wiki/OpenGL\#Specification

http://vr.isdale.com/WhatIsVR/frames/WhatIsVR4.1.html

Shuqiu, Z., Lei, L., Xinyu, G.: VRML Realization of Virtual Plants Growth. Journal of Capital Normal University (Natural Science Edition), 12 (2003)

Amditis, A., Karaseitanidis, I., Mantzouranis, I.: Virtual Reality Research in Europe: Towards Structuring the European Research Area, Product Engineering (2008)

Springer, S.L., Gadh, R.: State-of-the-art virtual reality hardware for computer-aided design. Journal of Intelligent Manufacturing 7(6), 12 (1996) 